\title{
Stoffwechsel und Wachstum bei Scyphomedusen
}

\author{
F. KRÜGER \\ Biologische Anstalt Helgoland, Zentrale, Hamburg 50
}

\begin{abstract}
Metabolism and growth in Scyphomedusae. Measurements on the rate of growth of Rbizostoma octopus conducted by THIEL (1966) yielded an unusual shape of the graphically plotted growth curve. The close relation between rates of growth and metabolism suggested, as a first step, the investigation of the oxygen consumption of this jelly-fish especially as a function of body weight. In accordance with the high rate of growth, oxygen consumption related to wet weight does not decrease during growth and shows weight proportionality. The same seems to be true for specimens of the related Cyanea and Chrysaora species. The rate of oxygen consumption of Scyphomedusae is rather high. Related to the organic matter the oxygen consumption in Rhizostoma amounts to $2077 \mathrm{~mm}^{3} / \mathrm{g} / \mathrm{h}$ at $15^{\circ} \mathrm{C}$. This value is much higher than in other invertebrates. The unusual growth curve of Rhizostoma octopus affords a revision of the growth types established by vON BERTALANFFY (1942). His third growth type cannor be distinguished exactly from the first type and hence must be united with it. The observations on Rbizostoma seem to indicate the existence of another third type, where the rate of growth increases with increasing body size.
\end{abstract}

\section{EINLEITUNG}

Vor kurzem veröffentlichte THIEL (1966) Daten über das Wachstum der Scyphomeduse Rhizostoma octopus. Diese Qualle tritt bei Helgoland normalerweise nur im Herbst mit einem Scheibendurchmesser von etwa 20 bis $30 \mathrm{~cm}$ auf. Daher nahm man bislang an, daß sie vom Kanal oder vom Atlantik angetrieben wird. Dr. H. KüHL von der Außenstelle der Bundesforschungsanstalt für Fischerei in Cuxhaven machte Herrn Dr. THIEL darauf aufmerksam, daß die Blumenkohlqualle in großer Zahl im Plankton von Cuxhaven in allen Größenstufen - angefangen bei den kleinsten Ephyren - auftritt. Wir müssen uns also von der Vorstellung befreien, daß diese Qualle einen Fremdling in unserer Fauna darstellt. Das Auftreten jüngster Ephyren, die bei einer längeren Verdriftung sicherlich metamorphosiert hätten, beweist das Vorkommen der zugehörigen Polypen im Bereich unserer Küste und insbesondere auch in der Nachbarschaft von Cuxhaven. Allerdings hat man bis jetzt noch nicht die zugehörigen Polypen finden können (KüHL 1964, 1967).

THIEL, der sich schon seit längerer Zeit für Wachstumsprobleme interessiert, nahm die hier gebotene günstige Gelegenheit wahr, Daten über das Wachstum dieser Meduse zu sammeln. Im Gezeitenstrom der Elbe tritt sie zeitweise in großer Zahl auf. Auf Grund meines eigenen Interesses für die mathematische Erfassung des tierischen Wachstums unternahm ich den Versuch, die von THIEL gegebenen Daten für das offensichtlich 
sehr starke Wachstum dieses Coelenteraten genauer zu analysieren. Die Medusen treten etwa Mitte Juni als Ephyren mit einem Scheibendurchmesser von weniger als $10 \mathrm{~mm}$ auf. Bis zum September wachsen sie zu einem Scheibendurchmesser von $250 \mathrm{~mm}$ und mehr heran. Danach scheinen die Medusen nach der Abgabe der Geschlechtsprodukte abzusterben. Ende September beobachtete ich sie nicht mehr in Helgoland.

Die Ablösung der Ephyren von den Polypen erfolgt offensichtlich nicht nur zu einem einzigen Zeitpunkt, sondern erstreckt sich bis in den August hinein, zu welcher Zeit - allerdings in geringerer Zahl - noch sehr kleine Medusen auftreten. In den späteren Fängen treten daher Medusen sehr unterschiedlicher Größe auf. Es heben sich bei den einzelnen Fängen aber deutlich bestimmte Größenklassen heraus. Die Zahlenangaben von ThIEL (1966) von 6 Fangterminen in der Zeit vom 25. Juni bis zum 3. September 1964 stellen Durchschnittswerte der einzelnen Fänge dar. Sie stimmen mit den Medianwerten überein und lassen sich auch in den Verteilungskurven deutlich als $\mathrm{Ma}$ xima erkennen. Da die Zeitschrift, in der die Arbeit publiziert wurde, nicht überall zugängig sein dürfte, halte ich es für nützlich, seine Tabelle 7, die seine Auswertungen zusammenfaßt, an dieser Stelle wiederzugeben (Tab. 1).

Tabelle 1

Daten von ThIEL (1966) uiber das Wachstum von Rbizostoma octopus

\begin{tabular}{|c|c|c|}
\hline \multirow[b]{2}{*}{ Fangtage } & \multicolumn{2}{|c|}{ Scheibendurchmesser } \\
\hline & $\begin{array}{c}\text { bei mehr als } 50 \% \text { der } \\
\text { Medusen }\end{array}$ & Gesamt-Mittelwerte \\
\hline 25.6. 1964 & 10 bis $30 \mathrm{~mm}$ & $18 \mathrm{~mm}$ \\
\hline 13.7 .1964 & 10 bis $40 \mathrm{~mm}$ & $28,3 \mathrm{~mm}$ \\
\hline 23.7 .1964 & 30 bis $60 \mathrm{~mm}$ & $48,8 \mathrm{~mm}$ \\
\hline 6. 8.1964 & 40 bis $90 \mathrm{~mm}$ & $65,3 \mathrm{~mm}$ \\
\hline 21. 8.1964 & 60 bis $130 \mathrm{~mm}$ & $94,0 \mathrm{~mm}$ \\
\hline 3. 9.1964 & 180 bis $270 \mathrm{~mm}$ & $225,2 \mathrm{~mm}$ \\
\hline
\end{tabular}

Die graphische Darstellung der linearen Werte für den Scheibendurchmesser in Abhängigkeit von den Fangdaten liefert eine außerordentlich steile Kurve. Interessanter und für Wachstumsanalysen aufschlußreicher ist - allgemein - die semilogarithmische Darstellung (relative Wachstumskurve), bei der man die Logarithmen der Meßwerte als Funktion des linearen Zeitwertes aufträgt. Hierbei ergab sich ein unerwartetes Bild. Zeichnet man die relative Wachstumskurve eines Fisches, einer Muschel oder eines Säugetieres, so erhält man eine Kurve, die sich zur Abszisse öffnet. Abweichend verhalten sich z. B. die Insekten, die bei dieser Art der Darstellung angenähert eine Reihe von Geraden liefern, welche durch die Häutungsprozesse unterbrochen sind. Die Wachstumsdaten von THIEl für Rbizostoma ergeben aber eine Kurve, deren Krümmungssinn dem der meisten Kurven entgegengesetzt ist (Abb. 1). Die Kurve verläuft im Anfangsteil weniger steil als später.

VON BertalAnFFy (1942) hat auf den Zusammenhang zwischen der Art der GröBenabhängigkeit des Stoffwechsels und der Form der Wachstumskurve hingewiesen. Es 
erschien daher von Interesse, die Größenabhängigkeit der Atmung bei Scyphomedusen und insbesondere bei Rhizostoma zu untersuchen.

Nicol (1967) gibt den Sauerstoffverbrauch von Rbizostoma bei $16^{\circ} \mathrm{C}$ mit 4,2 bis $11,7 \mathrm{~mm}^{3} / \mathrm{g} / \mathrm{h}$ bei einem Mittelwert von 7,2 $\mathrm{mm}^{3}$ an. Die älteren Messungen von WINTERSTEIN (1905) sind mit den vorliegenden Ergebnissen nicht zu vergleichen. Bei einer Versuchstemperatur von $12^{\circ} \mathrm{C}$ wird der Sauerstoffverbrauch mit etwa $7 \mathrm{~mm}^{3}$ angegeben.

Die von den früheren Autoren gemessenen Daten geben nur die Atmungsgröß3e je Gewichtseinheit an. Angesichts der in der Regel auftretenden Reduktion des Stoff wechsels im Verlauf des Wachstums ist diese Form der Angabe der Stoffwechselgröße un-

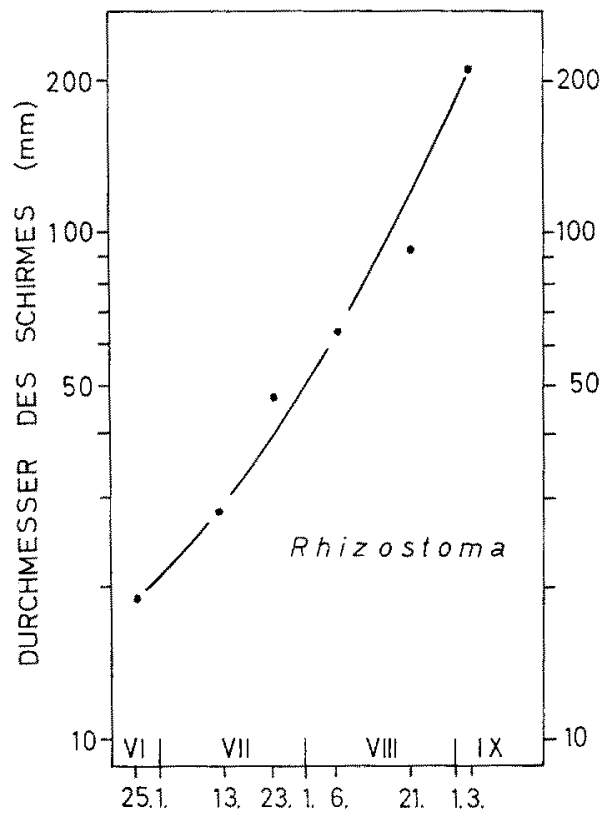

Abb. 1: Semilogarithmische Darstellung (relative Wachstumskurve) der Wachstumsdaten von THIEL (1966) für Rbizostoma octopus

zureichend. Eine für weitergehende Analysen brauchbare Beschreibung der Atmungsgröße eines Organismus erfordert unbedingt die Angabe des allometrischen Exponenten, der den Umfang der Stoffwechselreduktion beschreibt. Sie erfordert also die Auswertung der Messungen auf der Basis der allometrischen Funktion. Diese wiederum setzt die Bestimmung der Atmungsgröße einer Art über eine möglichst weite Gewichtsspanne voraus. PüTTER (1909) setzte für die Scyphomedusen die Gültigkeit der Oberflächenregel voraus. Nrcol macht hierüber keine Angaben, scheint sie aber auch anzunehmen. Der besondere Verlauf der Wachstumskurve von Rhizostoma octopus forderte aus dem oben genannten Grunde die Untersuchung der Größenabhängigkeit der Atmung dieser Meduse. Ich nahm bei dieser Untersuchung die Gelegenheit wahr, das gleiche Problem auch an Chrysaora bysoscella und Cyanea zu bearbeiten. 


\section{MATERIAL UND METHODE}

Den Kernpunkt der Untersuchung bildete Rhizostoma octopus. Die benötigten Medusen ließen sich recht leicht an der von THIEL angegebenen Stelle im Jachthafen von Cuxhaven sammeln. Man muß beim Arbeiten mit Medusen vermeiden, daß sie mit Luft in Berührung kommen oder mechanisch stärker beansprucht werden. Daher sind in einem Schleppnetz gefangene Medusen für physiologische Untersuchungen ungeeignet. Wir schöpften die mit dem Kescher gefangenen Tiere unter Wasser mit einem Schöpflöffel aus dem Netz und übertrugen sie vorsichtig in den bereitstehenden Behälter mit Brackwasser aus dem Hafen.

Dieser Fundort der Medusen ist dadurch bemerkenswert, daß es sich um ein im höchsten Grade verschmutztes Gewässer handelt. Es treiben dort nicht nur faulende Fische von den benachbarten Fischverwertungsbetrieben, sondern auch Holzspäne und Abfälle jeglicher Art, und auf der Oberfläche schwimmen zahlreiche Oiflecke. Erstaunlicherweise fand sich jedoch ein außerordentlich reiches Plankton in dem Kescher.

Die Messungen erfolgten im Laboratorium der Hamburger Zentrale der Biologischen Anstalt Helgoland. Die Tiere überstanden den Autotransport sehr gut und konnten im Konstanzraum bei $15^{\circ} \mathrm{C}$ bei bestem Wohlbefinden gehältert werden. Als Futter erhielten die Medusen im Mixer zerkleinertes Muschelfleisch, das auch Herr HoltmanN am Helgoländer Aquarium zur Fütterung benutzt. Zur Ergänzung führte ich in dem Helgoländer Laboratorium noch Atmungsmessungen an Chrysaora bysoscella und Cyanea durch, die dort leicht in verschiedenen Größenstufen zu beschaffen waren.

Die Atmungsmessungen erfolgten in der von mir beschriebenen Apparatur (KRÜGER 1960), die nach dem Durchströmungsprinzip arbeitet. Sie gestattet, von jedem Individuum eine Reihe von Meßdaten zu erhalten, deren arithmetisches Mittel den Auswertungen zugrunde liegt. Die Sauerstoffbestimmungen erfolgten nach der WinklerMethode.

Die Apparatur habe ich in der Zwischenzeit dadurch weniger anfällig gegen Bruchgefahr gemacht, daß die Mehrzahl der Leitungen jetzt aus Trovidur besteht. Dieser Kunststoff ist offensichtlich hinreichend undurchlässig für Gase, um keine Störungen zu verursachen. Nur die Temperier-Schlange, das Verteilerrohr und die Tierbehälter bestehen noch aus Glas. $\mathrm{Da}$ es sich in der Zwischenzeit zeigte, daß Polyäthylenschlauch für Sauerstoff merklich durchlässig ist, ersetzte ich ihn an den Verbindungsstellen durch dickwandigen Gummischlauch. Die Elastizität der angewandten Kunststoffleitungen gestattete überdies, die Zahl der Schlauchverbindungen wesentlich zu vermindern. Schlauchverbindungen befinden sich jetzt nur noch an den Ableitungen aus den Tierbehältern, die für das Auswechseln der Winklerflaschen eine gewisse Beweglichkeit besitzen müssen.

Die Tierbehälter waren genügend groß, daß die Medusen sich frei darin bewegen können. Um zu verhindern, daß die Versuchstiere durch den Wasserstrom gegen die Ausströmungsöffnung der Behälter gedrückt werden und damit die Messungen verhindern, stellte ich in die Behälter kleine Gestelle aus Kunststoff, die oben eine Platte besaßen, die kurz unter der Ausströmungsöffnung der Behälterwandung anlag, durch eine Reihe von Bohrungen aber dem Wasser ungehinderten Durchtritt ließ. Die ganz großen Medusen, die nicht in die vorhandenen Tierbehälter paßten, untersuchte ich in einem 
Filtriertopf nach WITT, in den die notwendigen Leitungen eingebaut waren. In diesem Falle übertrug ich die Medusen mit Hilfe von wassergefüllten Plastiktaschen in den Behälter, der einen Durchmesser von $20 \mathrm{~cm}$ hatte.

Bei den Messungen an Rbizostoma in Hamburg kam Seewasser zur Anwendung, das um die Hälfte mit Leitungswasser verdünnt war. Hierdurch stellte ich etwa den Salzgehalt des Brackwassers im normalen Biotop her. Durch ihr Vorkommen im Mündungsgebiet der Elbe dürfte Rbizostoma gegen Schwankungen des Salzgehaltes nicht sehr empfindlich sein. Die Messungen an den großen Exemplaren von Rbizostoma erfolgten im Helgoländer Laboratorium. In diesem Falle konnte ich nur normales Seewasser benutzen. Das gleiche war auch der Fall bei den Messungen an den anderen Medusenarten.

Die Temperatur betrug bei allen Messungen $15^{\circ} \mathrm{C}$. Nach dem Prinzip der gesteuerten Kühlung (KRÜGER 1957) war sie auch bei höherer Raumtemperatur leicht exakt einzuhalten. Eine Ausnahme bilden nur die Messungen in dem WITtschen Topf, für den kein genügend großer Thermostat zur Verfügung stand. In diesem Falle konnte die Versuchstemperatur nur näherungsweise eingestellt werden.

Nach Abschluß der Messungen ließ ich auf einer Glasplatte das den Medusen anhaftende Seewasser ablaufen und brachte sie in passende Wägegläser, die mit Fließpapier ausgelegt waren, dessen Trockengewicht vorher bestimmt wurde. Nach der Bestimmung des Frischgewichtes, das naturgemäß gewisse Unsicherheiten einschließt, wurden die Medusen zur Bestimmung der Trockensubstanz bei etwa $100^{\circ} \mathrm{C}$ bis zur Gewichtskonstanz getrocknet. Auch diese Bestimmung kann durch die Hygroskopie der Calcium- und Magnesiumchloride nicht ganz exakt ausfallen. Es entstehen aber nur relative Fehler, die alle Wägungen gleichmäßig betreffen.

Da bei den Medusen die Trockensubstanz im wesentlichen aus den Salzen des Meerwassers besteht, versuchte ich auch den Gehalt an organischer Substanz zu bestimmen. $\mathrm{Zu}$ diesem $\mathrm{Zweck}$ wurde die Trockensubstanz auf dem Filter mit einer $5 \%$ Formol-Lösung ausgewaschen und zweimal mit destilliertem Wasser nachgewaschen, um die löslichen Salze zu entfernen. Anschließend wurde wieder getrocknet. Ich benutzte eine Formol-Lösung zum Auswaschen der Salze, um die Eiweiß-Substanzen in eine unlösliche Form zu überführen und hierdurch zu verhindern, daß sie mit in Lösung gehen. Bei den kleinsten Medusen versagte das Verfahren, da der Gehalt an organischer Substanz zu gering war.

\section{ERGEBNISSE DER ATMUNGSMESSUNGEN}

Angesichts der Tatsache, daß sich in der Stoff wechselphysiologie die Gültigkeit der allometrischen Funktion zur Beschreibung der Gewichtsabhängigkeit von Prozessen in zahllosen Fällen bewährt hat und die einzige Möglichkeit für eine einwandfreie Beschreibung der Versuchsergebnisse darstellt, erfolgte die Auswertung der Meßergebnisse auf ihrer Basis. In logarithmierter Form lautet diese Gleichung:

$$
\log y=\log b+a \cdot \log w
$$

In dieser Form stellt die allometrische Funktion die Gleichung einer Geraden dar, und deshalb erhalten wir bei der Darstellung unserer Meßergebnisse in einem doppelt loga- 
rithmisch unterteilten Koordinatensystem eine Gerade, in deren Nähe die Meßpunkte angeordnet sind. Die Unschärfe biologischer Meßwerte bedingt naturgemäß eine mehr oder minder starke Streuung um die Regressionsgerade, welche die exakte Funktion darstellt.

Die graphische Darstellung bietet nicht nur einen einfachen und schnellen Weg, um schon im Verlauf der Experimente eine Übersicht über die Versuchsergebnisse zu bekommen, sondern gestattet auch näherungsweise die Bestimmung der beiden in der Gleichung enthaltenen Parameter $b$ und $\alpha$. Der Parameter $\alpha$ stellt nämlich in der Gleichung den Tangens des Steigungswinkels der Regressionsgeraden dar. Messen wir mit einem Winkelmesser die Steigung der Regressionsgeraden, so können wir in einer Tabelle der Winkelfunktionen den zugehörigen Wert für $\alpha$ ablesen. Im allgemeinen schwankt der Wert von $\alpha$ zwischen 0,6 und 1,0. Ein Wert von 0,66 entspricht einer oberflächenproportionalen Atmung; der Steigungswinkel beträgt in diesem Falle etwa 340. Eine gewichtsproportionale Atmung wird durch den $\alpha$-Wert 1,0 beschrieben; der zugehörige Steigungswinkel beträgt $45^{\circ}$. Höhere $\alpha$-Werte werden durch einen stärkeren Kurvenanstieg gekennzeichnet.

Neben dem Exponenten können wir auch den Wert für $b$ aus der graphischen Darstellung ablesen. Wie sich aus mathematischen Gründen leicht erkennen läßt, stellt dieser Wert die Stoffwechselgröße eines Individuums von der Einheit des gewählten Gewichtes dar. Wir suchen also auf der Abszisse, auf der man normalerweise die unabhängige Variable - in unserem Falle also das Gewicht - abträgt, den Wert 1 auf und finden den zugehörigen Schnittpunkt mit der Regressionsgeraden. Dieser Schnittpunkt stellt den Parameter $b$ dar. In der Literatur wird der $b$-Wert oft als bedeutungslos bezeichnet. Dadurch aber, daß er die auf die Gewichtseinheit bezogene Stoffwechselgröße beschreibt, bildet er eine wertvolle Basis für Vergleichszwecke. Bei der allometrischen Auswertung stellt $b$ das geometrische Mittel der Meßwerte dar. Bei der Berechnung aus den linearen Daten würde sich ein etwas höherer Wert ergeben. Die graphische Darstellung bietet eine einfache Möglichkeit zur Kontrolle der mathematisch exakten Berechnung.

Da biologische Meßwerte stets eine gewisse Schwankung um einen zugrunde liegenden Mittelwert zeigen, gestatten wenige Meßpunkte keine zuverlässige Bestimmung der Parameter der allometrischen Funktion. Will man zu zuverlässigen Ergebnissen kommen, so muß man die Gewichtsspanne der Versuchstiere so weit wählen, daß die Abweichungen der Einzelmessungen vom theoretischen Wert gering werden gegenüber den Differenzen, die sich aus dem gesamten Kurvenverlauf ergeben. Aus diesem Grunde wertete ich bei meinen Versuchen an Rbizostoma eine Spanne von nahezu 4 Zehnerpotenzen aus, nämlich Medusengewichte im Bereich zwischen 0,1 g und mehr als $1000 \mathrm{~g}$. Bei der recht großen Schwankungsbreite der Einzelwerte erwies sich diese Weite der Spanne auch als erforderlich. Die graphische Darstellung der Parameter der allometrischen Funktion ergänzte ich durch die rechnerische Auswertung nach der üblichen Form der Regressionsberechnung. Hierbei ist allerdings zu beachten, daß einzelne an den Enden des Meßbereiches liegende Werte, die stärkere Abweichungen von der Funktion zeigen, das rechnerische Ergebnis stark beeinflussen und dadurch zu irreführenden Werten führen können. Hier bietet die graphische Darstellung ein wertvolles Hilfsmittel, solche Werte zu erkennen. Sie anzuwenden sollte daher nie versäumt werden. Die in neuerer 
Zeit von Morphologen vorgeschlagene Berechnung der reduzierten Hauptachse scheint mir für physiologische Probleme keinen Vorteil zu bieten.

Die doppelt logarithmische Darstellung der Meßwerte für den Sauerstoffverbrauch. von Rhizostoma octopus in Abhängigkeit vom Feuchtgewicht ist in Abbildung 2 wiedergegeben. Schon der Augenschein läßt den starken Anstieg der Atmungswerte mit dem Gewicht erkennen, der offensichtlich in der Nähe von $45^{\circ}$ liegt. Ein klein wenig aus

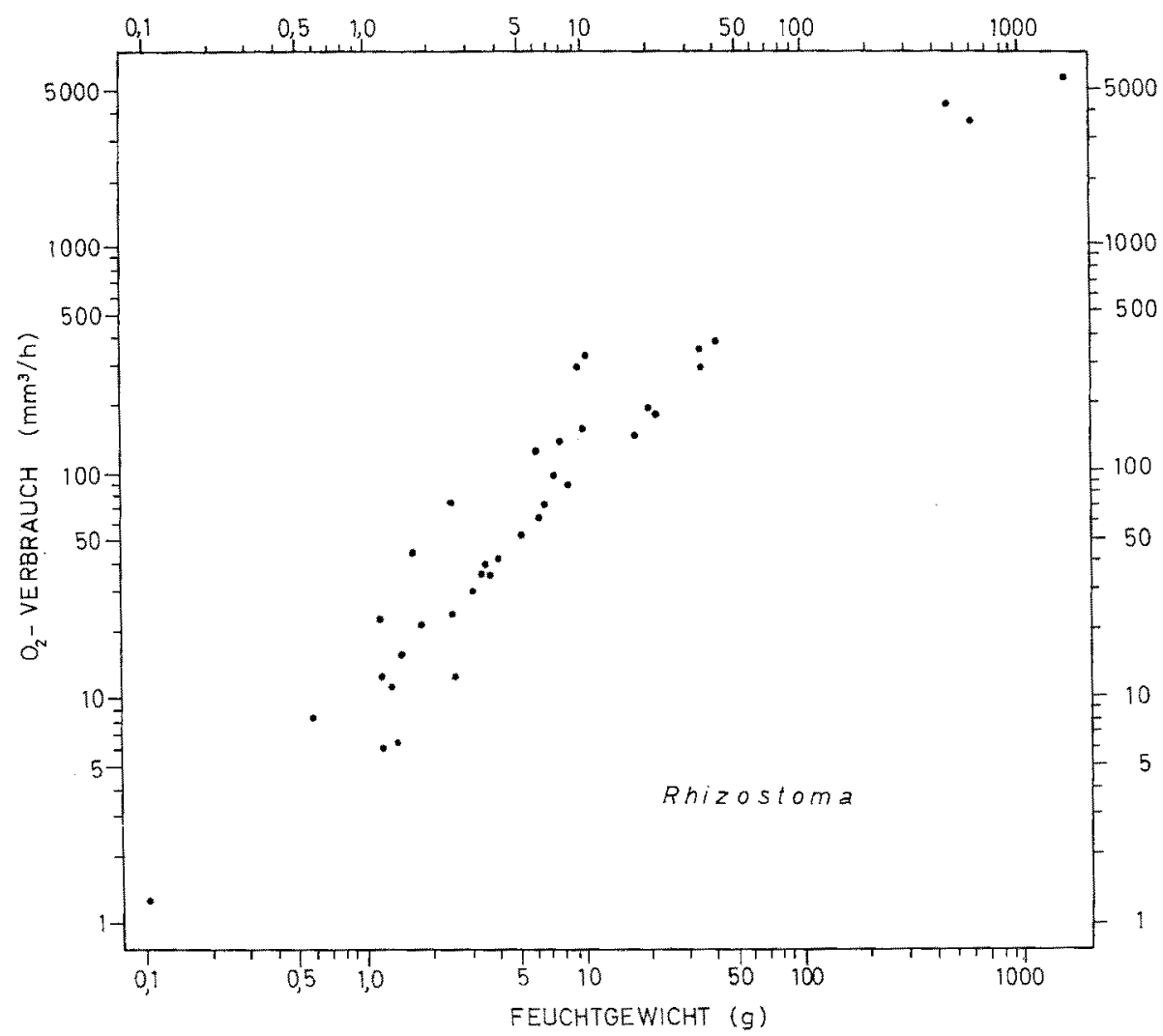

Abb. 2: Doppellogarithmische Darstellung der Meßwerte für den Sauerstoffverbrauch von Rbizostoma octopus in Abhängigkeit vom Feuchtgewicht

dem Verlauf der übrigen Punkte fallen die Werte für die drei schwersten Medusen heraus, ohne ihn aber grundsätzlich zu verändern. Hierbei ist aber zu berïcksichtigen, daß die Messungen an diesen Medusen nicht ganz unter den gleichen Bedingungen erfolgten, wie bei den übrigen Tieren. Zum einen war die Salzkonzentration bei diesen Messungen höher, und zum andern konnte die Temperatur nicht ganz exakt eingestellt werden. Läßt man aus diesem Grunde die drei Messungen bei der Berechnung aus, so erhält man für den Exponenten $\alpha$ rechnerisch den Wert von 0,988 - praktisch also gleich 1,0. Dieses Ergebnis war aus der graphischen Darstellung zu erwarten. Die Atmung von Rbizostoma octopus verläuft also - bezogen auf das Feuchtgewicht - gewichtsproportional. Der Sauerstoffverbrauch je Einheit des Feuchtgewichtes wird ausgedrückt durch den 
Wert $b$; er beträgt $13,5 \mathrm{~mm}^{3} / \mathrm{g} / \mathrm{h}$. Es entspricht dieser Verbauch etwa dem oberen Grenzwert, den NicoL (1967) angibt.

Die überraschende Tatsache, daß Rhizostoma octopus eine gewichtsproportionale Atmung zeigt, scheint auch für die beiden anderen untersuchten Scyphomedusen zuzutreffen, wenn auch in diesem Falle aus technischen Gründen die Sicherung des allo-

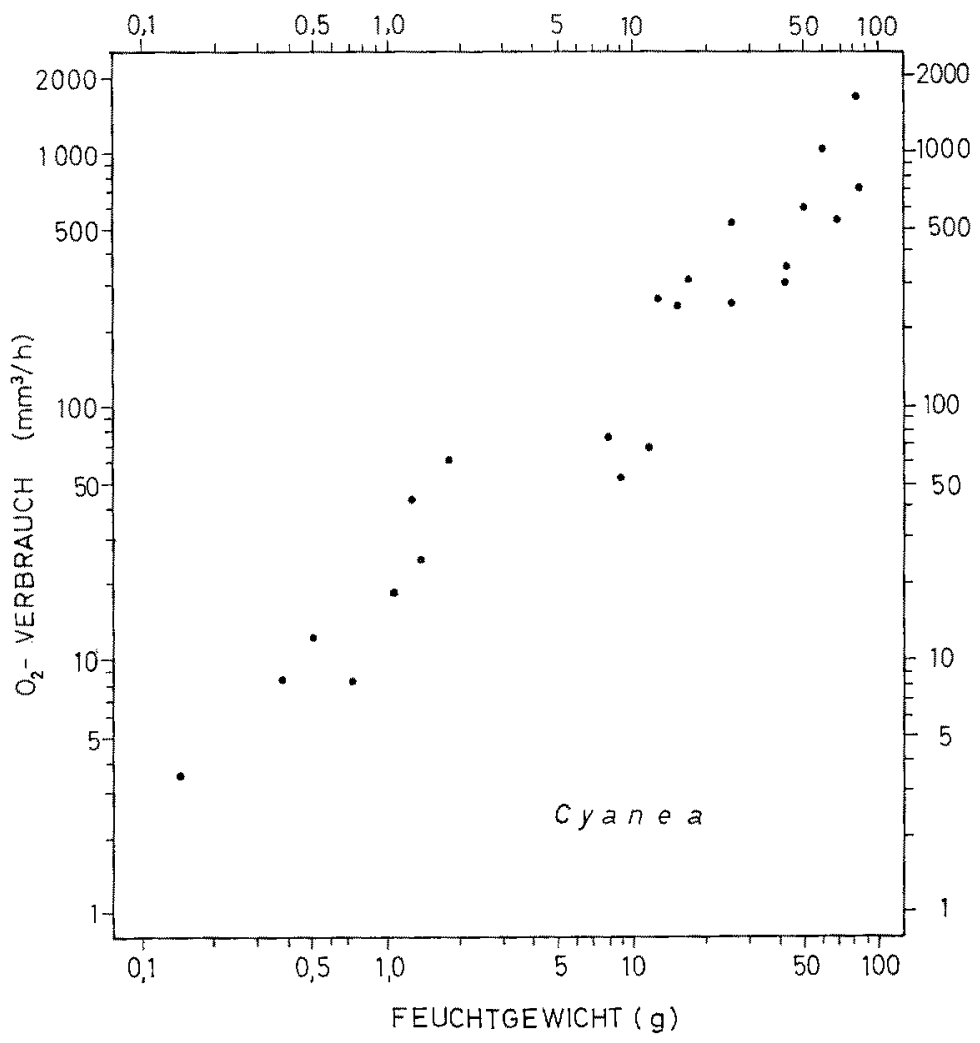

Abb. 3; Doppellogarithmische Darstellung der Meßwerte für den Sauerstoffverbrauch von Cyanea als Funktion des Feuchtgewichtes

metrischen Exponenten nicht mit der gleichen Signifikanz möglich war. Ausschlaggebend war hierbei der Umstand, daß bei beiden Medusen nur eine geringere Gewichtsspanne erfaßt werden konnte. Die erhaltenen Werte zeigen aber bei Cyanea wie auch bei Chrysaora eindeutig, daß der Wert des allometrischen Exponenten nicht mit einer oberflächenproportionalen Atmung vereinbar ist.

Abbildung 3 zeigt den sehr starken Anstieg der Meßpunkte für die Atmung von Cyanea. In diesem Falle konnte eine Gewichtsdifferenz von 3 Zehnerpotenzen erfaßt werden; rechnerisch ergibt sich für den allometrischen Exponenten $\alpha$ der Wert 0,91. Hierbei besteht allerdings der Verdacht, daß zwei verschiedene Cyanea-Arten zur Untersuchung kamen, nämlich C. lamarckii und C. capillata, die ich bei den Messungen nicht unterscheiden konnte. Die Trennung der beiden Arten glückte erst in neuerer Zeit durch die Untersuchung von THIEL (1962) auf Grund der relativen Tentakelzahlen. 
Beide Arten kommen bei Helgoland vor. Legt man als allometrischen Exponenten den Wert 1,0 zugrunde, so kommt man für Cyanea zu einem Sauerstoffverbrauch von $15,8 \mathrm{~mm}^{3} / \mathrm{g} / \mathrm{h}$. Er ist also merklich höher als bei Rhizostoma.

Die Messungen an Chrysaora bysoscella lassen ebenfalls den steilen Anstieg des Sauerstoffverbrauchs mit zunehmender Größe erkennen (Abb. 4). Material und Zeitmangel hinderten mich leider, die Atmungswerte bei Individuen über $500 \mathrm{~g}$ Gewicht durch weitere Meßwerte zu sichern. Berïcksichtigt man nicht den vollkommen aus den

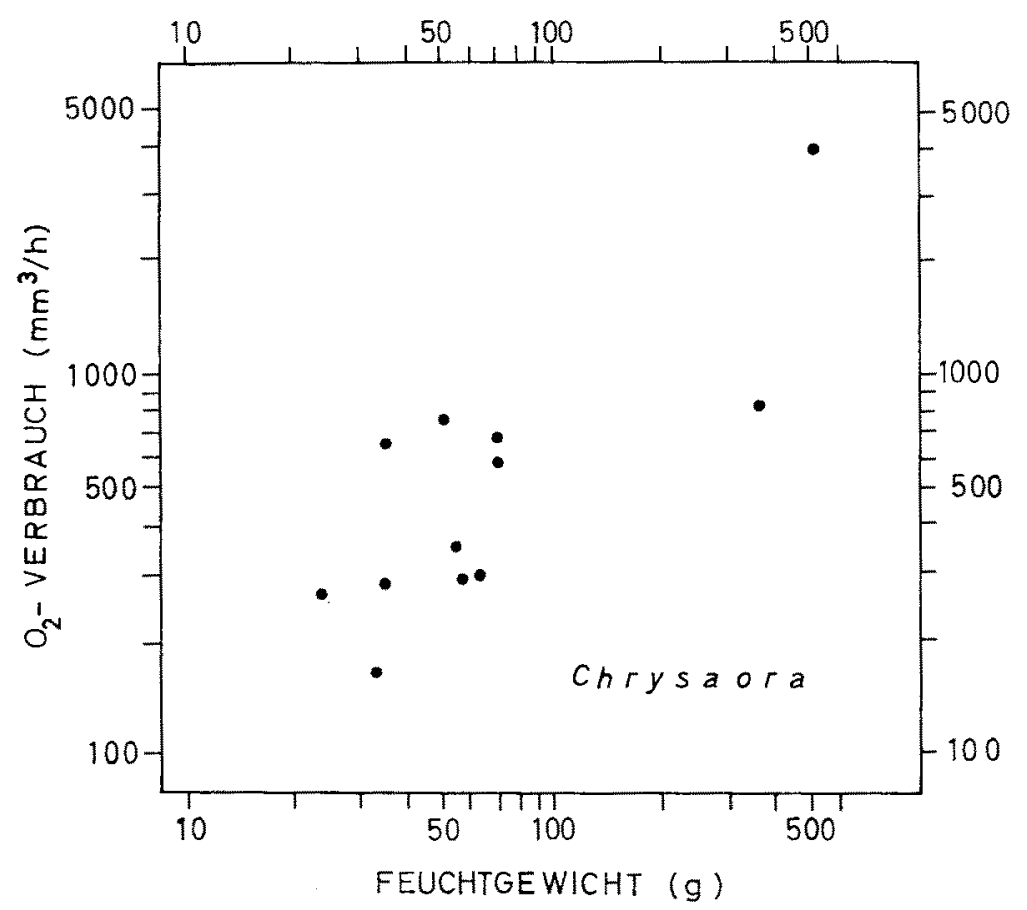

Abb. 4: Doppellogarithmische Darstellung der Meßwerte für den Sauerstoffverbrauch von Cbrysaora hysoscella als Funktion des Feuchtgewichtes

übrigen Werten herausfallenden Sauerstoffverbrauch der Meduse von $324 \mathrm{~g}$ Gewicht, bei der eine Schädigung bei der Übertragung in den Atmungsapparat nicht ausgeschlossen werden konnte, so ergibt sich aus den übrigen Daten ein $\alpha$-Wert von 0,98 , also ebenfalls eine gewichtsproportionale Atmung. Nennenswert niedriger liegt dagegen bei Chrysaora der auf die Gewichtseinheit bezogene Sauerstoffverbrauch von $6,6 \mathrm{~mm}^{3} / \mathrm{h}$.

\section{BETRACHTUNGEN ZUR ATMUNGSINTENSITAT DER SCYPHOMEDUSEN}

Die in diesen Versuchen nachgewiesene Proportionalität zwischen Sauerstoffverbrauch und Gewicht bei Scyphomedusen muß als vollkommen unerwartet bezeichnet werden. Pütrer (1909) nahm gerade wegen der starken Oberflächenentwidklung der 
Medusen eine oberflächenproportionale Atmung an. Wir haben es bei den Medusen mit Organismen zu tun, die eine ansehnliche Größe erreichen. Ihr Vaskularsystem ist zwar sehr gut entwickelt und nach den Beobachtungen von THIEL von einem starken Wasserstrom durchspült. Er dringt aber nicht in den Schirm ein, der die Hauptmasse der Tiere bildet. Dieser ist auf eine Sauerstoffversorgung durch Diffusion angewiesen. Es dürfte unwahrscheinlich sein, daß der ganze Sauerstoffverbrauch konzentriert ist auf die Außenflächen der Medusen und das direkt vom Wasserstrom durchspülte Gastrovaskularsystem. Zur eindeutigen Klärung dieser Frage wären Messungen an den einzelnen Organsystemen erforderlich. Bei der sehr starken Zunahme der Körperanhänge der Scyphomedusen im Verlauf ihres Wachstums wäre eventuell an eine massenproportionale Vergrößerung der Gesamtoberfläche zu denken. Immerhin wäre in diesem Falle eine außerordentlich starke Konzentrierung der Atmungsaktivität in der Oberfläche zu erwarten. Diese ist aber schon, wie weiter unten gezeigt wird, uberraschend hoch. Daher erscheint mir die Möglichkeit nicht wahrscheinlich.

Gerade bei der relativ wenig aktiven Rhizostoma hatte ich einen sehr niedrigen Sauerstoffverbrauch vermutet und war über seine Größenordnung erstaunt. Ein Sauerstoffverbrauch won beinahe $16 \mathrm{~mm}^{3} / \mathrm{g} / \mathrm{h}$ liegt durchaus in der Größenordnung der Atmung anderer Evertebraten. Berechnet auf das Feuchtgewicht verbraucht eine Miesmuschel von $1 \mathrm{~g}$ Gewicht etwa $70 \mathrm{~mm}^{3} / \mathrm{g} / \mathrm{h}$ bei der gleichen Temperatur (KRüGer 1960). In ähnlicher Größenordnung liegt auch der Sauerstoffverbrauch von Arenicola (KRÜGER 1964). Da bei diesen beiden Beispielen der Sauerstoffverbrauch mit zunehmender Größe erheblich abnimmt, verschiebt sich beim Vergleich schwerer Individuen das Verhältnis zugunsten der Medusen.

Die unerwartet hohe Atmungsintensität der Scyphomedusen wird noch augenscheinlicher, wenn man den Vergleich nicht auf der Basis des Feuchtgewichtes vornimmt, sondern ihn auf den Gehalt an organischer Substanz bezieht. Dieser ist bei den Medusen bekanntlich außerordentlich gering. Ich versuchte, ihn an den untersuchten Individuen zu bestimmen. Der geringe Gehalt an organischer Substanz ließ bei der angewandten Technik nur Bestimmungen an Medusen mittlerer Größe zu. Bei den kleinen Medusen war die Grenze durch die Empfindlichkeit der Waagen gegeben, bei den großen $\mathrm{Me}$ dusen konnte ich wegen der Kürze der zur Verfügung stehenden Zeit nicht die für eine exakte Bestimmung erforderliche Homogenisierung durchführen.

Für Rbizostoma liegen die meisten Daten vor. Zwischen einem Feuchtgewicht von 3,5 bis $39 \mathrm{~g}$ nimmt der Gehalt an Trockensubstanz in bester Näherung proportional zum Gewicht zu und errechnet sich zu 2,96\%. Der Wassergehalt dieser Art würde sich demnach auf $97 \%$ belaufen. Der relative Gehalt an organischer Substanz ändert sich zwischen 8 und $39 \mathrm{~g}$ nicht (Abb. 5) und errechnet sich im Mittel zu 0,53\%. Der Gehalt an löslichen Salzen würde demnach 2,43\% betragen. Da die untersuchten Rhizostomen aus dem Brackwasser stammten, war ein Wert dieser Größenordnung zu erwarten. Leider unterlief bei den entsprechenden Bestimmungen an Cyanea und Chrysaora ein technischer Fehler, so daß sie nicht zuverlässig auszuwerten waren. Der Gehalt an Trockensubstanz und organischer Substanz scheint höher zu liegen.

Nehmen wir den Gehalt an organischer Substanz bei Rbizostoma mit $0,53 \%$ an und stellen ihn in Beziehung zu dem theoretischen Sauerstoffverbrauch einer $100 \mathrm{~g}$ schweren Meduse, so würde dieser $1350 \mathrm{~mm}^{3} / \mathrm{h}$ betragen. Auf die Gewichtseinheit 
organischer Substanz bezogen ergibt sich ein Sauerstoffverbrauch von $2547 \mathrm{~mm}^{3}$. Die Miesmuschel enthält etwa $15 \%$ Trockensubstanz. Hiervon sind 3\% etwa als Salz abzuziehen. Der Gehalt an organischer Substanz beträgt demnach etwa $12 \%$ des Frischgewichtes. Nimmt man für eine Miesmuschel von $1 \mathrm{~g}$ Gewicht als Durchschnittswert einen Sauerstoffverbrauch von $70 \mathrm{~mm}^{3}$ an, so ergibt das je Gewichtseinheit organischer

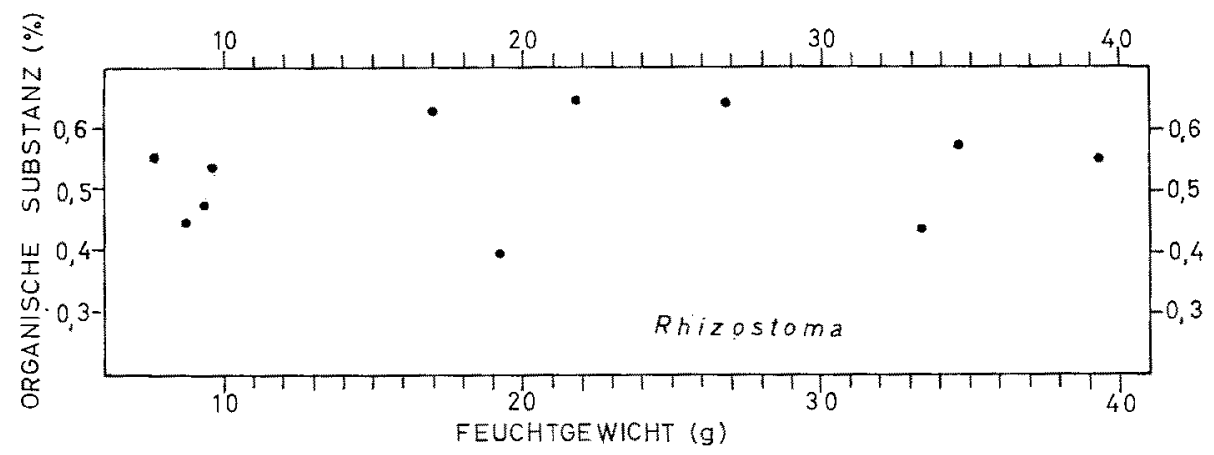

Abb. 5: Lineare Darstellung des prozentualen Gehaltes an organischer Substanz bezogen auf das Feuchtgewicht

Substanz einen Sauerstoffyerbrauch von $583 \mathrm{~mm}^{3}$. Mit zunehmender Größe nimmt bei der Miesmuschel der Sauerstoffverbrauch sehr stark $a b$, so daß sich die Verhältnisse noch mehr zu ihren Ungunsten verschieben. Dieser Bezug auf die organische Substanz macht die unerwartet hohe Stoffwechselintensität der Scyphomedusen noch deutlicher. Es ist nicht anzunehmen, daß sie aus der - gerade bei Rbizostoma-geringen Aktivität resultiert, sondern daß sie einen Ausdruck für den hohen Wachstumsstoffwechsel darstellt.

\section{ZUR FRAGE DER WACHSTUMSTYPEN VON VON BERTALANFFY}

Den Ausgangspunkt dieser Untersuchungen bildete die aus den Beobachtungen von THIEL (1966) sich ergebende hohe Wachstumsintensität von Rbizostoma octopus. Nach der Modellvorstellung von von Bertalanffy $(1934,1942)$ mußte eine so hohe Wachstumsintensität ihren Ausdruck in der Form der Größenabhängigkeit des Stoffwechsels finden. Diese Vermutung konnte im Prinzip auch durch die vorliegenden Meßergebnisse bestätigt werden, sowohl hinsichtlich der absoluten Größe des Sauerstoffverbrauchs, wie auch durch den hohen Wert für den allometrischen Exponenten $\alpha$.

Von BERTALANFFY hat bekanntlich nach der Größenabhängigkeit des Stoffwechsels drei Wachstumstypen unterschieden. Der erste wird durch eine oberflächenproportionale Atmung (allometrischer Exponent $=0,66$ ) gekennzeichnet, den zweiten Typus bilden die Beispiele einer gewichtsproportionalen Atmung (allometrischer Exponent $=1,0$ ). Den dritten Typus bilden die Fälle, in denen der allometrische Exponent der Atmung zwischen 0,66 und 1,0 liegt. Dem ersten Typus schreibt von BERTALANFFy einen Wachstumsverlauf zu, der bei linearer Darstellung eine kontinuierlich sich abflachende Kurve 
liefert. Im Gegensatz hierzu soll der dritte Typus durch eine Sigmoid-Kurve für das Längenwachstum ausgezeichnet sein. Diese beiden Typen sind aber nicht voneinander zu trennen, denn gerade die Fische, auf die sich vON BERTALANFFy für die Aufstellung des ersten Typus beruft, zeigen in der Mehrzahl der Fälle einen allometrischen Exponenten von etwa 0,8, wären demnach also dem dritten Typus zuzuordnen. Überdies kennen wir auch Kurven für das Längenwachstum von Fischen, die ein Maximum zeigen, also S-förmig sind. Der dritte Wachstumstyp von von BERTALANfFy kann daher nicht aufrechterhalten werden.

Aufrechterhalten bleiben muß aber der zweite Typus, bei dem der Stoffwechsel Masse-proportional verläuft, wie es bislang für die Insekten und anscheinend auch für die terrestrischen Pulmonaten bekannt war. In linearer Darstellung zeigt das Insektenwachstum eine Kurve, deren Krümmung entgegengesetzt gerichtet ist, die wir bei Fischen, Säugetieren und Mollusken finden. Für den zweiten Typus benutzt von BERTALANFFY zur Abgrenzung die Gerade, die sich bei semilogarithmischer Darstellung ergibt. Es ist aber nicht zulässig, Wachstumstypen auf Grund grundsätzlich verschiedener Kurvendarstellungen aufzustellen. Wir müssen in allen Fällen entweder die lineare oder die semilogarithmische Darstellung vergleichen. BERTALANFFys erster und zweiter Typus zeigen bei dieser Art der Darstellung den gleichen Krümmungssinn - ein in der linearen Darstellung eventuell vorhandener Wendepunkt fällt fort. Die steil aufwärts gekrümmte lineare Wachstumskurve der Insekten wird in semilogarithmischer Darstellung in guter Näherung zu einer Geraden, wenn man von den Unstetigkeiten absieht, welche die Häutungen bedingen. Hierdurch unterscheidet sich der zweite Typus von von BertalanfFy eindeutig vom ersten Typus und muß erhalten bleiben. Als dritter Typus würde nun nach den Daten von T'HIEL der über das exponentielle Wachstum der Insekten hinausgehende Fall von Rhizostoma octopus als logische Ergänzung hinzutreten.

Der erste Typus, der offensichtlich am weitesten verbreitet ist, läßt sich oberhalb des Wendepunktes zum Beispiel durch die BertalanfFY-Funktion beschreiben. Besser geeignet ist aber die Funktion von Zucker (1941) und von mir (KRÜGer 1965), die auch den Wendepunkt von Wachstumskurven erfaßt. Ihre prinzipielle Formulierung lautet

$$
y=a^{-\frac{1}{\tau}}
$$

Das Insektenwachstum wird durch die Zinseszinsformel beschrieben:

$$
y=a \cdot b^{x}
$$

Die Wachstumsdaten von ThIEL (1966) für den dritten Typus lassen sich in befriedigender Weise durch eine Funktion wiedergeben, die dem Prinzip der GoMpertz-Formel entspricht:

$$
y=a^{b^{\tau}}
$$

Bei dieser Funktion ergibt sich durch doppelte Logarithmierung der Meßwerte Proportionalität zum linearen Zeitwert. Wie Abbildung 6 zeigt, entsprechen die Zahlen von THIEL recht gut dieser Forderung. Hierbei bleibt es aber offen, ob die gewählte Formulierung endgültig ist, da sie sich nicht mit der allometrischen Funktion in Einklang bringen läßt. 
In den Formeln stellt der Parameter a für den zweiten und dritten Wachstumstypus eine Anfangsgröße dar. In beiden Fällen erscheint das Wachstum mathematisch nicht begrenzt, wie schon voN BERTALANFFY für das Insektenwachstum betonte. In der Formulierung des ersten Typus stellt der Parameter a eine Endgröße dar, auf die auch von Bertalanffy seine Wachstumsdarstellung bezieht. Der Parameter $b$ stellt in allen drei Formeln eine Geschwindigkeitskonstante dar.

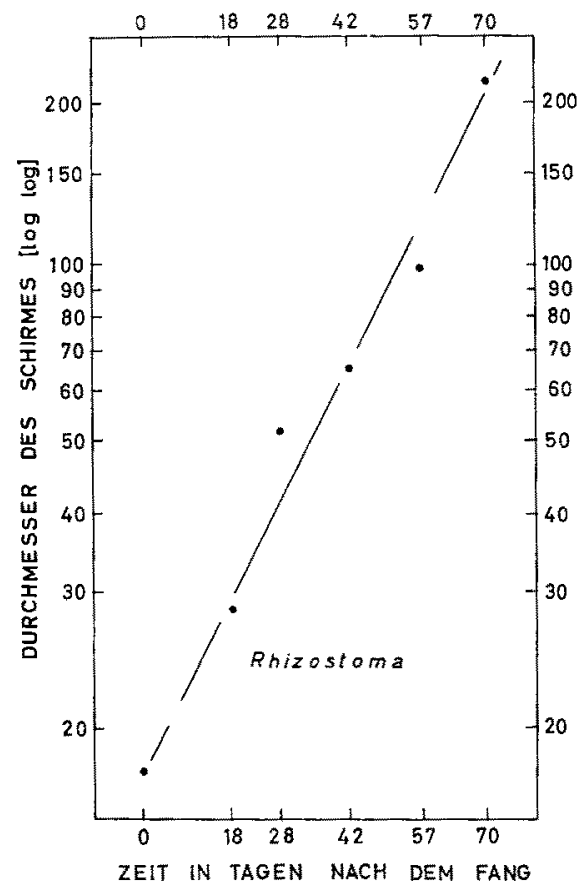

Abb. 6: Darstellung der log log-Werte für den Scheibendurchmesser von Rhizostoma octopus als Funktion des linearen Alterswertes

In der hier vorgeschlagenen Form, die den drei Wachstumstypen einheitlich die semilogarithmische Darstellung zugrunde legt, ergibt sich ein folgerichtiges System (Abb, 7), das in gleicher Weise die Einsetzung von Längen- und Gewichtsdaten oder anderen Dimensionen gestattet. Die erhaltenen Kurven unterscheiden sich nur hinsichtlich ihrer Steigung, ermöglichen aber immer in einfacher Weise eine Zuordnung zu einem der drei Typen.

Der dritte übersteile Typus wird vorläufig allerdings nur durch die isoliert stehende Zahlenreihe von THIeL (1966) für Rbizostoma octopus gestützt. THIEL hat auch an anderen Scyphomedusen Wachstumsmessungen vorgenommen, die allerdings noch kein abschließendes Urteil gestatten. Seine Daten für Aurelia aurita (1959) beruhen auf nur zwei Probenahmen. Er versucht sie durch ältere Beobachtungen von Browne (1901) zu ergänzen. Da die Möglichkeit von Unterschieden in den Umweltbedingungen nicht auszuschließen sind, die das Wachstum beeinflußten, scheint mir ein solches Vorgehen nicht statthaft. Die von THIEL (1960) gegebenen Daten für das Wachstum von Cyanea capil- 
lata sind für eine exaktere Auswertung ungeeignet. Die Streuung der Meßwerte ist sehr breit, und es heben sich keine bevorzugten Größenklassen ab. Unter diesen Umständen hat eine Mittelwertbildung nur sehr bedingten Wert. Die aus seinen Daten errechneten arithmetischen Mittel ergeben eine Kurve des - normalen - ersten Typus. In diesem Falle wäre er aber mit einer gewichtsproportionalen Atmung verknïpt, wie die Messungen ergaben.

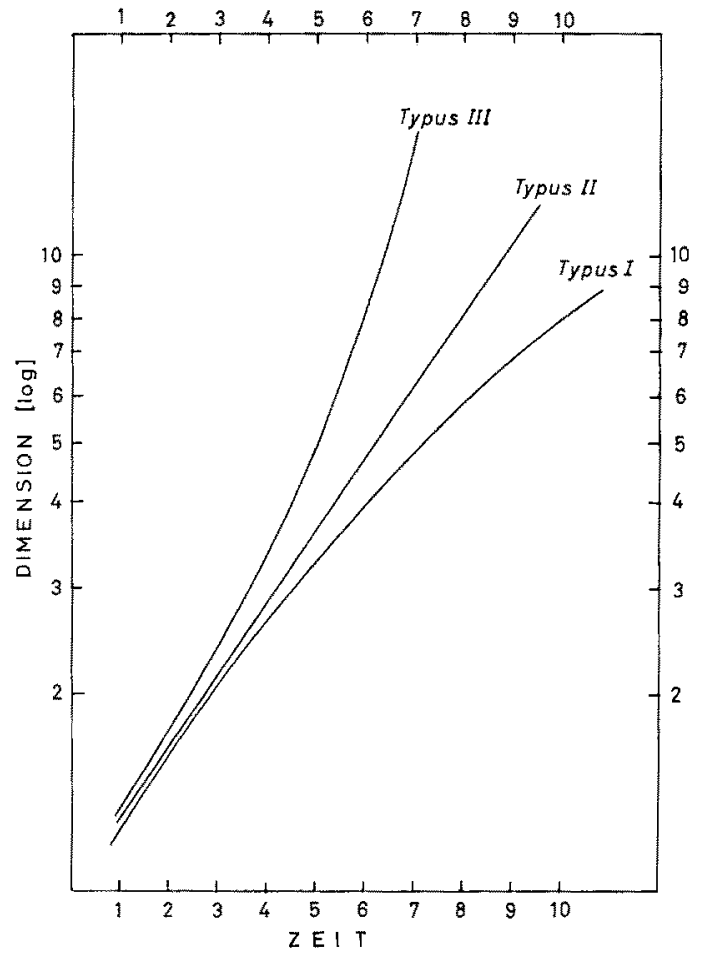

Abb. 7: Schematische Darstellung für den Verlauf der semilogarithmischen Wachstumskurven für die drei unterschiedenen Wachstumstypen

Man könnte angesichts des außergewöhnlichen Verlaufs der Wachstumskurve von Rhizostoma octopus denken, daß sie durch die Umweltbedingungen verursacht wird. Unter ihnen könnte die Temperatur eine Rolle spielen. ThIEL hat schon 1963 einige Proben von Rbizostoma gesammelt. Vergleicht man die Mittelwerte dieser Proben mit den Werten des Jahres 1964, die dieser Arbeit zugrunde gelegt wurden, so ergeben sich wesentlich geringere Zahlen. Vermutlich wirkt sich in dieser geringeren Wachstumsrate der vorausgegangene sehr strenge Winter aus, durch den bis in den Juni hinein die Oberflächen-Temperaturen des Seewassers in der Elbmündung erheblich unter den normalen Werten lagen. Diese tiefen Wassertemperaturen dürften die Ablösung der Ephyren verzögert haben.

Unter diesen Umständen könnte man daran denken, daß die abnorme Wachstumskurve von Rbizostoma octopus dadurch entsteht, daß mit steigender Wassertemperatur die Wachstumsgesdhwindigkeit in den Sommermonaten gesteigert und hier- 
durch der starke Anstieg der Wachstumsrate verursacht wird. Nun liegen die Verhältnisse bei Rbizostoma dadurch günstig, daß das ganze Wachstum in den Sommermonaten zwischen Juni und September abläuft, zu einer Zeit also, zu der der Kälteeinfluß des Winters abgeklungen ist, und während der nur eine relativ geringfügige Temperaturerhöhung im August erfolgt. Die Oberflächentemperaturen in der Elbmündung im Jahr 1964 betrugen: im Juni $13,3^{\circ} \mathrm{C}$, im Juli $16,2^{\circ} \mathrm{C}$, im August $17,0^{\circ} \mathrm{C}$ und im September $15,6^{\circ} \mathrm{C}$. Es wäre ungewöhnlich, wenn diese geringen Temperaturdifferenzen einen so starken Einfluß auf das Wachstum von Rhizostoma ausüben würden.

\section{DAS PROBLEM DER ERNAHRUNG VON RHIZOSTOMA}

Der unerwartet hohe Sauerstoffverbrauch von Rbizostoma octopus wirft die Frage auf, in welcher Weise sie den hiermit zwangsweise verbundenen hohen Nahrungsbedarf deckt. Schon Pütrer (1909) erkannte dieses Problem. Bei dieser Gattung ist die weite Mundöffnung der Scyphomedusen in eine große Zahl feiner Kanäle aufgeteilt, die eine Aufnahme größerer Beutetiere ausschließen. ThIEL (1964) bringt eine ausführliche Zusammenstellung der Literatur über das Problem der Ernährung von Rbizostoma. Experimentell konnte er zwar nachweisen, daß mit dem starken Wasserstrom in den Saugkanälen Beuteobjekte in der Größe von Copepoden in das Gastrovaskularsystem aufgenommen werden können. Trotzdem erscheint fraglich, ob auf diese Weise der Nahrungsbedarf der Meduse gedeckt wird. PüTTER versucht ihn dadurch anschaulich zu machen, daß er die Zahl der Copepoden berechnet, die zur Deckung des Bedarfs erforderlich wären und ermittelt ihn für eine Meduse von $232 \mathrm{~g}$ Gewicht zu 24000 Copepoden. Im allgemeinen findet man aber keine geformte Nahrung im Gastrovascularsystem von Rhizostoma. Allenfalls wurden wenige Copepoden, Amphipoden und Isopoden nachgewiesen, aber nicht in der Menge, die PÜTTER errechnete. Rbizostoma gehört aus diesem Grunde zu den Beispielen, die er für seine Lehre von der Ernährung der Wassertiere durch gelöste Substanzen heranzieht. Die Möglichkeit der Aufnahme gelöster organischer Substanzen durch Meerestiere scheint nach neuesten Ergebnissen von STEPHENs (1966) nicht von der Hand zu weisen sein, wie mit radioaktiv markierten Substanzen gezeigt werden konnte.

Es wäre aber auch daran zu denken, daß Rhizostoma Detritus, Nannoplankton und Bakterien als Nahrungsquelle ausnutzt. Diese Frage bleibt - wie noch manch andere - zu klären.

\section{ZUSAMMENFASSUNG}

1. Die graphische Auswertung der Wachstumsdaten von THIEL (1966) für Rhizostoma octopus ergab eine sehr hohe Wachstumsrate. Die semilogarithmische Wachstumskurve zeigt einen ganz ungewöhnlichen Verlauf (Abb. 1). Der Zusammenhang zwischen Wachstum und Stoffwechsel legte die Untersuchung der Größenabhängigkeit des Sauerstoffverbrauchs dieser Scyphomeduse nahe.

2. In Ubereinstimmung mit der hohen Wachstumsrate ergab sich für Rbizostoma octopus eine gewichtsproportionale Atmung. 
3. Auch für Chrysaora bysoscella und Cyanea konnte eine gewichtsproportionale Atmung wahrscheinlich gemacht werden.

4. Berechnet man den Sauerstoffverbrauch von Rhizostoma octopus auf die organische Substanz, die nur etwa $0,53 \%$ des Feuchtgewichtes ausmacht, so kommt man zu dem außergewöhnlich hohen Wert von $2547 \mathrm{~mm}^{3} / \mathrm{g} / \mathrm{h}$ bei $15^{\circ} \mathrm{C}$.

5. Die von VON BERTALANFFY (1942) aufgestellten Wachstumstypen werden diskutiert. Sein III. Typus ist gegen Typus I nicht abzugrenzen; er muß deshalb aufgegeben werden.

6. Der Verlauf der semilogarithmischen Wachstumskurven bietet eine geeignete Basis für eine folgerichtige Unterscheidung von Wachstumstypen. Hiernach ergibt sich folgendes Bild: I. Ty p u s: Die Wachstumskurve öfnet sich zur Abszisse, das heißt, die Wachstumsrate nimmt mit zunehmendem Alter ab. (Häufigster Typus, bei Wirbeltieren, Mollusken etc.) II. T' y p u s : Die Wachstumskurve wird durch eine Gerade gebildet, das heißt, die Wachstumsrate bleibt konstant. III. T y p u s : Der Krimmungssinn der Wachstumskurve ist entgegengesetzt dem von Typus I, das heißt, die Wachstumsrate nimmt mit zunehmender Größe zu (vorläufig nur durch die Daten für Rbizostoma belegt).

Es ist nicht möglich, alle Angehörigen der Anstalt zu nennen, die mich bei der Durchführung dieser Untersuchung unterstützten. Hervorheben möchte ich nur, die interessierte Mitarbeit von Frl. M. BAUER, die mit großem Verständnis die Messungen durchführte und die Tierbeschaffung durch Herrn W. KRüss in Helgoland - aud in Zeiten von Materialknappheit. Herrn Dr. KüHL von der Außenstelle Cuxhaven der Bundesforschungsanstalt für Fischerei bin ich für die Mithilfe und Beratung bei der Materialbeschaffung in Cuxhaven zu großem Dank verpflichtet.

\section{ZITIERTE LITERATUR}

BerTAlanffy, L. V., 1934. Untersuchungen über die Gesetzlichkeit des Wachstums. Allgemeine Grundlagen der Theorie. Arch. EntwMech. Org. 131, 813-653.

- 1951. Theoretische Biologie. Bd 2. Stoffwechsel, Wachstum. (1. Aufl. 1942. Borntraeger, Berlin, 362 pp.) 2. Aufl. Francke, Bern, 418 pp.

Browne, E. T., 1901. Variation in Aurelia aurita. Biometrica 1, 90-108.

KRÜGER, F., 1957. Temperatur-Regelung durch gesteuerte Kühlung. Helgoländer wiss. Meeresunters. 6, 71-75.

-- 1960. Zur Frage der Größenabhängigkeit des Sauerstoffverbrauchs von Mytilus edulis. Helgoländer wiss. Meeresunters. 7, 125-148.

- 1964. Versuche über die Abhängigkeit der Atmung von Arenicola marina von Größe und Temperatur. Helgoländer wiss. Meeresunters. 10, 38-63.

- 1965. Zur Mathematik des tierischen Wachstums. I. Grundlagen einer neuen Wachstumsfunktion. Helgoländer wiss. Meeresunters. 12, 78-136.

KüHL, H., 1964. Die Scyphomedusen der Elbmündung. Veröff. Inst. Meeresforsch. Bremerh. 9, 84-94.

- 1967. Scheibenquallen an unseren Küsten. Mikrokosmos 56, 169-174.

Nicol, J. A. C., 1967. The biology of marine animals. 2nd ed. Pitman \& Sons, London, $699 \mathrm{pp}$.

PÜTTER, A., 1909. Die Ernährung der Wassertiere und der Stoffhaushalt der Gewässer. Fischer, Jena, $168 \mathrm{pp}$. 
STEPHENS, G. C., 1966. Uptake of organic material of aquatic invertebrates. IV. The influence of salinity on the uptake of amino acids by the brittle star, Opbiactis arenosa. Biol. Bull. mar. biol. Lab., Woods Hole 131, 172-185.

THIEL, M. E., 1959. Beiträge zur Kenntnis der Wachstums- und Fortpflanzungsverhältnisse von Aurelia aurita. Abb. Verh. naturw. Ver. Hamburg 3, 13-26.

- 1960. Beobachtungen über Wachstum, Variationen und Abnormitäten bei der Cyanea capillata der Ostsee. Abh. Verh. naturw. Ver. Hamburg 4, 89-108.

- 1962. Untersuchungen zur Artfrage von Cyanea lamarckii und Cyanea capillata. Abb. Verh. naturve. Ver. Hamburg 6, 277-293.

- 1964. Untersuchungen über die Ernährungsweise und den Nahrungskreislauf bei Rhizostoma octopus. Mitt. hamburg. zool. Mus. Inst. 61 (Erg.Bd), 247-269.

- 1966. Untersuchungen über die Herkunft, das Auftreten, das Wachstum und die Fortpflanzung von Rhizostoma octopus im Elbmündungsgebiet. Abb. Verh. naturre. Ver. Hamburg $10,59-88$.

Winterstein, H., 1905. Wärmelähmung und Narkose. Z. allg. Physiol. 5, 323-350.

ZUCKER, L. \& ZUCKER, T. F., 1941. A simple time weight relation in well nourished rats. J. gen. Physiol: 25, 445-463. 